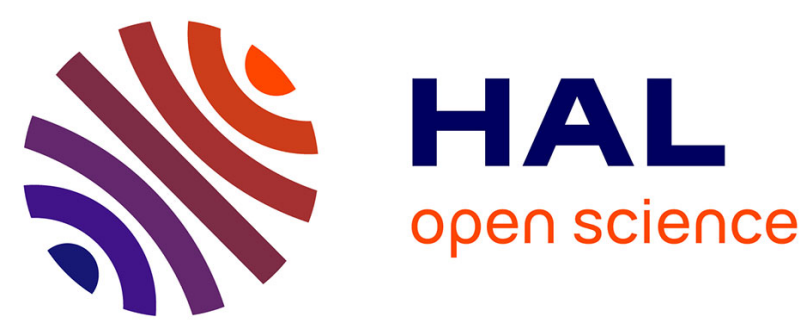

\title{
Diabetes and Blood Glucose Disorders Under Anti-PD1
}

Quentin Magis, Caroline Gaudy-Marqueste, Agnès Basire, Anderson Loundou, Nausicaa Malissen, Laura Troin, Sandrine Monestier, Stephanie Mallet, Sylvie Hesse, Marie-Aleth Richard, et al.

\section{- To cite this version:}

Quentin Magis, Caroline Gaudy-Marqueste, Agnès Basire, Anderson Loundou, Nausicaa Malissen, et al.. Diabetes and Blood Glucose Disorders Under Anti-PD1. Journal of Immunotherapy, 2018, epub ahead of print. 10.1097/CJI.0000000000000218 . hal-01763824

\section{HAL Id: hal-01763824 \\ https://hal-amu.archives-ouvertes.fr/hal-01763824}

Submitted on 11 Apr 2018

HAL is a multi-disciplinary open access archive for the deposit and dissemination of scientific research documents, whether they are published or not. The documents may come from teaching and research institutions in France or abroad, or from public or private research centers.
L'archive ouverte pluridisciplinaire HAL, est destinée au dépôt et à la diffusion de documents scientifiques de niveau recherche, publiés ou non, émanant des établissements d'enseignement et de recherche français ou étrangers, des laboratoires publics ou privés. 
1 Title: Diabetes and blood glucose disorders under anti-PD1

2

3 Quentin Magis ${ }^{1}$, Caroline Gaudy-Marqueste ${ }^{1}$, Agnes Basire ${ }^{3}$, Anderson Loundou ${ }^{4}$,

4 Nausicaa Malissen ${ }^{1}$, Laura Troin ${ }^{1}$, Sandrine Monestier ${ }^{1}$, Stéphanie Mallet ${ }^{1}$, Sylvie

5 Hesse $^{1}$, Marie-Aleth Richard ${ }^{1}$, René Valéro², Sophie Beliard ${ }^{2}$, Jean-Jacques Grob ${ }^{1}$

7 (1) Aix-Marseille university, UMR 911, INSERM CR02, Dermatology and skin cancer

8 department, Hôpital de la Timone, APHM, Marseille, France

9 (2) Aix-Marseille university, Endocrinology department, Hôpital de la Conception, 10 APHM, Marseille, France

11 (3) Etablissement Français du Sang, Marseille, France

12 (4) Public health department, Aix-Marseille university, APHM, Marseille, France 13

14 Corresponding author:

15 Pr Caroline Gaudy-Marqueste

16 Dermatology and skin cancer department.

17264 rue Saiint Pierre

1813385 Marseille Cedex 5

19 France

20 Phone number : 0033491388591

21 Email : caroline.gaudy @ap-hm.fr

22

23 Funding : none

24 Conflict of interest: none

25 Word count : 2627

26 Abstract : 260

27 Figures : 3

28 Tables : 4

29 


\section{Abstract}

Acute type 1 diabetes (AD1) is a rare but definitive immune related adverse event associated with anti-PD1. Most of reported cases are close to what has been described as "fulminant type 1 diabetes". We sought to determine wheither anti-PD1 could impair glycoregulation and weither occurrence of AD1 could be anticipated by prior glycemic changes. Fasting glycaemia collected before, under and after treatment in melanoma patients treated with anti-PD1 over a period of 36 months were retrospectively analyzed. Glycemic trend analyses was performed using linear regression analysis. 1470 glucose values were monitored in 163 patients treated for a mean duration of 5.96 months. Three patients developed an AD1 (1, 84\%). Two other cases were observed in the same period in a still blinded trial of anti-PD1 vs Ipilimumab. All cases of AD1 occured in patients with a normal pretreatment glycaemia and there was no detectable drift of glycaemia prior to ketoacidosis onset. In 4 of the 5 cases of AD1, HLA subgroups were DRB01* 03 or 04 known to increase type 1 diabetes risk in general population.In the 28 patients with preexisting type 2 diabetes, there was a slight trend for glycaemia increase with anti-PD1 infusions (0.05 $\mathrm{mmol} / \mathrm{L} /$ infusion $\mathrm{p}=0.004)$. In the 132 patients with normal pretreatment glycaemia, there was a slight trend for a decrease of glycaemia with anti-PD1 infusions ($0.012 / \mathrm{mmol} / \mathrm{L} /$ infusion $\mathrm{p}=0.026)$. These data suggest that the monitoring of glycaemia under anti-PD1 cannot help to anticipate AD1, and there is no general tendency to glycemic disorder. HLA-genotyping before treatment may help to focus surveillance in patients with the HLA DRB1*03/04 group.

Key words: anti-PD1, melanoma, diabetes, glycaemia, immune related adverse 56 event, HLA

57

58

59

60

61

62

63 


\section{INTRODUCTION}

The development of new immunotherapies with check point inhibitors including antiCTLA4 antibodies and more recently anti-PD1 antibodies has dramatically improved the prognosis of metastatic melanoma [1-5]. Anti-PD1 have also demonstrated efficacy in many other malignancies like non-small cell lung cancer, renal cell carcinoma, head and neck squamous cell carcinoma and relapsing Hodgkin lymphoma.

Many immune-related adverse events (IRAE) have been reported with checkpointinhibitors including dermatitis, enterocolitis, hepatitis, thyroiditis, hypophysitis as well as some less frequent but potentially life threatening rare AEs [6,7]. In the context of an increasing number of patients exposed to these drugs, management of these IRAEs has become a priority and specific guidelines have been established [6]. While no warning signal for diabetes induced by checkpoint inhibitors has been detected during the clinical trials, several cases of acute insulin-dependent type 1 diabetes (AD1) have been recently reported under anti-PD1, and anti- PDL1 antibodies [8-10], and more recently under the anti-CTLA4 and anti-PD1 combination [11].

The exact mechanisms of these acute insulin-dependent diabetes under anti-PD1 therapies is currently unknown. Evidence that blockade of PD1-PDL1 checkpoint can accelerate the emergence of autoimmune diabetes in the non-obese diabetic mousemodel [12] suggests it may play a role in protecting against the development of autoimmune diabetes. Anti-Glutamic Acid Decarboxylase (GAD) autoantibodies or Insulin auto antiboides (IAA) have been identified in approximatively half of the anti-PD1 induced AD1 [7,8,11,17-21]. Several reported cases share close similitudes with the "fulminant type 1 diabetes" frequent in East Asia [22]: i) abrupt onset of ketoacidosis, ii) low $\mathrm{HbA} 1 \mathrm{c}$ value despite a high plasma glucose level iii) absence of insulin secretion capacity after glucagon test. This brutal onset suggests a drastic immune reaction against $\beta$-cell.

Apart from "fulminant diabetes", a case of diabetic ketoacidosis with insulin requirement has also been reported in a patient who had preexisting type 2 diabetes controlled with metformin [17], suggesting that some patients with preexisting type 2 diabetes might become more difficult to equilibrate under antiPD1. 
In order to determine whether anti-PD1 could impair glycoregulation in more patients than expected, especially in those with preexisting type 2 diabetes, and whether AD1 could be anticipated by prior glycaemic changes we retrospectively analyzed blood glucose samples of a series of 163 consecutive patients treated by anti-PD1 antibodies for melanoma.

\section{PATIENTS AND METHODS}

We performed a single institution, descriptive study of consecutive patients treated with anti-PD1 for melanoma in the department of dermato-oncology of CHU Timone in Marseille, FRANCE since September 2013. Each patient gave is written consent. Data were recorded for each patient between the first anti-PD1 infusion and the date of final analysis on May 2016. Available fasting blood glucose values were collected from the hospital files and retriewed from external laboratories before (in the year preceding the treatment with anti-PD1), under (usually 24-72h before each infusion), and within the year after anti-PD1 discontinuation for those in whom it was discontinued. The following variables were also collected: initial and per-treatment weight changes, BMI (body mass index); personal or familial history of type 1 or type 2 diabetes; personal history and history of autoimmune disease; previous treatment with Ipilimumab (yes or no), type of anti-PD1 administred (Nivolumab or Pembrolizumab), dosage, number of infusions, and cumulative dose of exposure; treatment efficacy (partial or complete response, stable disease or disease progression); date and cause of anti-PD1 discontinuation. The World Health Organization (WHO) definition was used for the diagnosis of diabetes i.e. fasting plasma glucose $\geq 7.0 \mathrm{mmol} / \mathrm{L}[23]$.

\section{Statistical analysis}

Statistical analysis was performed using IBM SPSS Statistics version 20 (IBM SPSS Inc., Chicago, IL, USA). Continuous variables are expressed as means $\pm S D$ or as median with range ( $\min , \max$ ), and categorical variables are reported as count and percentages. When the distribution of differences between pairs was non-normally distributed, the Wilcoxn signed-rank test was used to compare pre and post glycaemia measurements. Glycemic trend analyses were performed using linear regression 
132 analysis. All the tests were two-sided. The statistical significance was defined as $133 \mathrm{p}<0.05$.

134

135

136

\section{RESULTS}

\section{Population and treatment}

139 The characterisics of the 163 consecutive patients treated with anti-PD1 over a period 140 of 36 months are presented in table 1. Twenty-eight patients (17.2\%) had at least one elevated pre-treatment glycaemia. Twelve of these 28 patients had a known history of type 2 diabetes whereas 16 were not known to be diabetic. No patient

143 had a previous history of type 1 diabetes. Ninety-five patients (64\%) were treated with 144 Nivolumab and 68 (36\%) with Pembrolizumab, 27 of which in the context of therapeutic 145 trials. All patients, but $3(1.8 \%)$ who were treated in the adjuvant setting, had a 146 metastatic melanoma. Treatment was first-line in 109 (66.9\%) patients. Fifty-four 147 (32.9\%) had previously been treated with ipilimumab. A total of 1920 infusions (1146 148 Nivolumab and 774 Pembrolizumab) were administered over the study period. The 149 mean duration of the anti-PD1 treatment was $4.5(0.5-40)$ months. Mean number of 150 anti-PD1 infusions was 12.1 (1-53).

151 At the time of datalock, study treatment had been discontinued in 97 (59.5\%) patients 152 for disease-related death in 34 (35.1\%), disease progression in 44 (45.4\%), complete 153 response in 8 patients (8.2\%), IRAEs in 7 patients (7.2\%), and other adverse events 154 non considered as IRAEs in 4 patients (4.1\%) (Bilateral lower-limb ischemia in 1, 155 dyspnea worsening in 1, intestinal ischemia in 1, and septic shock in 1 patient).

\section{Fasting glycaemia in the whole cohort}

158 Blood glucose samples were available in 160 of the 163 patients, and a total of 1470 159 before, under and after-treatment glycaemia were collected. There was a non160 significant trend toward a decrease of glycaemia with anti-PD1 infusions (-0.012 $161 \mathrm{mmol} / \mathrm{L} /$ infusions $\mathrm{p}=0.656)$. The median of the glycaemia did not change over the time 162 of anti-PD1 exposure. The evolution of the median (min, max) glycaemia according to 163 the number of anti-PD1 infusion is presented in figure1. 
164 The patients weight did not significantly change under treatment $(71.8 \mathrm{~kg}+/-15.1 \mathrm{vs}$ $16571.6 \mathrm{~kg} \mathrm{+} /-15.1, \mathrm{p}=0.429$, for mean pre-treatment and last available weight, 166 respectively).

167 There was no difference in mean fasting glycaemia values according to the anti-PD1 168 molecule administred (Nivolumab or Pembrolizumab) nor to the fact that they had 169 received ipilimumab before (data not shown).

171 Patients with normal glycaemia before-treatment

172 Among the 132 patients without known preexisting type 2 diabetes, and having normal 173 glycaemia before treatment, 3 (2.22\%) developed an AD1, all fullfilling the "fulminant 174 diabetes" criteria. Figure 2 represents the evolution of median (min, max) glycaemia 175 with successive anti-PD1 infusion in this population.

176 Apart from these $3 \mathrm{AD1}$, only one patient had one isolated increased glycaemia 177 (8.7mmol/L) under anti-PD1 therapy, which may correspond to inadequate non-fasting 178 sampling. In the 132 patients with normal glycaemia before treatment, there was a 179 statistically significant negative but very low trend toward a decrease of glycaemia ($180 \quad 0.012 / \mathrm{mmol} / \mathrm{L} /$ infusion $(p=0.026)$ ). Five patients received systemic corticosteroids $181(1 \mathrm{mg} / \mathrm{kg}$ ), for IRAE management (colitis $\mathrm{n}=2,1$ skin rash $\mathrm{n}=1$ ) or for symptomatic reason ( 2 cerebral edema).

\section{Patients with abnormal glycaemia before treatment}

185 Twelve patients had a preexisting treated type 2 diabetes, and 16 others had pretreatment fasting glycaemia compatible with type 2 diabetes definition (table 2). Out of the 16 who were previously untreated, only one patient required the introduction of repaglinide, whereas dietetic measures were sufficient to maintain glycaemia within the normal range in the 15 others. The 12 patients with a diagnosis of type 2 diabetes before treatment were respectively treated with insulin $(n=5)$, oral hypoglycemic agents $(n=15)$ (metformine (4), repaglinide (4), gliclazide (3), vidagliptine-metformine (1), sitagliptine-metformine (1), sitagliptine (1), glimepiride (1)). Among these 28 patients, $8(28.6 \%)$ had at least one elevated glycaemia (>10 $\mathrm{mmol} / \mathrm{L})$ under anti-PD1

194 therapy. Evolution of median glycaemia in this population is represented in figure 3.

195 Hemoglobin A1c (HbA1c) values were available only in 7 of these 28 patients. An $196 \mathrm{HbA1C}>7 \%$ was found in 2 patients during anti-PD1 treatment course, while their 197 weight remained stable. No type 2 patient not requiring insulin at baseline 
subsequently required insulin to manage hyperglycemia. Glycemic trend analysis by linear regression analysis suggested a slight increase of blood glucose values along with increasing number of infusions $(0.05 \mathrm{mmol} / \mathrm{L} /$ infusion $\mathrm{p}=0.004)$. Three patients received systemic corticosteroids for IRAE management (1 colitis) or symptomatic reason ( 2 cerebral edema).

\section{New onset AD1 under anti-PD1}

205 Three cases (1.84\%) of AD1 were diagnosed in the cohort: one after 2 doses of 206 Pembrolizumab, and the two others after 4 and 11 doses of Nivolumab respectively. 207 Two additional cases ( $\mathrm{n}^{\circ} 4$ and 5 ) were diagnosed in patient receiving immunotherapy 208 in blinded therapeutic trials (Ipilimumab versus Anti-PD1). One of them ( $\left.{ }^{\circ} 4\right)$ have 209 since been unblinded and confirmed having reveived nivolumab. As patient 5 is 210 still blinded, we are not certain that he received anti-PD1. A summary of these 5 cases

211 is provided in Table 3. Briefly, all of them presented a cardinal syndrome with 212 diabetic ketoacidosis, normal or subnormal HbA1C levels (range 6.4 to $7.6 \%$ ), 213 and collapsed C-peptide secretion. Two patients had slightly positive anti islet 214 antigen-2 antibodies. Four patients carried a predisposition HLA DRB1*03 or 215 HLA DRB1*04 haplotypes. Insulin therapy was initiated insulin therapy upon 216 presentation for all patients, and they all remained insulin-dependent at this 217 time.

\section{DISCUSSION}

221 This is the first systematic study of glycaemia in patients treated by anti-PD1 in the real 222 life setting. It does not support the idea that anti-PD1 could systematically induce 223 glycemic disorders or make preexisting diabetes more difficult to manage despite a 224 small trend for an increase of glycaemia along with anti-PD1 infusions. However, our 225 data confirm the possibility of anti-PD1-induced AD1, and suggest that incidence of 226 AD1 (1.8\% in this series) could be underestimated. The glycaemia monitoring shows 227 that AD1 cannot be anticipated by any preliminary drift in glucose metabolism. Our 228 data also suggest that some HLA group (HLA DRB1*03/04) may be a risk marker for 229 anti-PD1 induced AD1 in the Caucasian population. 
231 More than 25 cases of type 1 acute diabetes, most of them diagnosed in patients

232 treated for a melanoma, have been reported so far in the literature, 22 under anti-PD1,

2331 under anti-CTLA4 and anti-PD1 combination and 3 under anti-PDL1 [7-9,11,17-

234 21,24-30]. (Table 4). Among these reported cases, 3 patients had a personal history

235 of autoimmune thyroiditis, like 2 of our patients, and 8 had previously been treated with

236 ipilimumab $[7,17,18,20,29,31]$.

237

238 The five cases of AD1 described herein fulfill the criteria of " fulminant diabetes"[22]: a

239 sudden onset of hyperglycemia with ketoacidosis, normal or subnormal $\mathrm{HbA} 1 \mathrm{C}$ levels

240 and collapsed C-peptide secretion reflecting an absence of insulin-secreting capacity.

241 In the Japanese population where fulminant diabetes was described, it was suspected

242 to result from a rapid destruction of $\beta$-pancreatic cells secondary to a viral pancreatic

243 infection [32,33] on a genetic predisposed background (DRB1 * 0405-DQB1 * 0401),

$244[34,35]$. In the present study, no previous change in glycaemia was predictive, making

245 the anticipation of this complication impossible, a characteristic that justifies the term

246 fulminant.

248 In the general population the annual incidence of AD1 is estimated 0.1 to 36/ 100.000 249 [36-38]. In our cohort, 2\% of patients developed an AD1 under a mean period under 250 treatment of 4 months, which suggests a huge incidence increase 100 to 1000 times 251 higher compared to basic risk.

253 Our anti-PD1 AD1 cases lack the HLA haplotypes identified in the fuminant diabetes 254 described in the Asian population. However, it is noteworthy that 4 of our 5 pts carried 255 a HLA DRB $1{ }^{*} 03$ or HLA DRB $1{ }^{*} 04$ haplotypes known to be associated with a life-time 256 risk of AD1 3 to 5 times higher than in the general population and even 20 to 40 times 257 higher in patients carrying both the HLA DR3 and DR4 haplotypes [39]. As these 258 haplotypes were mentioned in several other Caucasian cases of AD1 under anti-PD1, 259 HLA DRB1*03 and 04 genotyping can be suspected to be a risk marker for AD1 in patients treated by anti-PD1. These data are compatible with the hypothesis that anti261 PD1 could trigger AD1 in genetically predisposed patients, who would have been 262 natural candidate to AD1 later on. The delay between the introduction of 263 immunotherapy and the onset of AD1 ranged from one week to 12 months, suggesting 264 that, when the genetic background is there, anti-PD1 can trigger the disease very fast. 
265 It is noteworthy that developing an AD1 under anti-PD1 does not seem to be in itself a

266 guarantee of successful treatment, although some results suggest some link between

267 response to anti-PD1 and occurrence of IRAE [40]. Tumor response was documented

268 in only 10 of the previous published cases. Some degree of response was observed in

2698 of these 10 patients, as well as in 4 of our 5 cases.

272 When focusing on patients with a known preexisting diabetes, or at least pretreatment 273 increased glycaemia compatible with a type 2 diabetes, we found a estimated pre274 treatment prevalence of diabetes at $17.1 \%$ for a mean age of 65.2 years, which is quite 275 similar to the prevalence in the French epidemiological study OBEPI [41]. Linear 276 regression analysis in these patients suggests a slight increase $(0.05 \mathrm{mmol} / \mathrm{L}$ per 277 infusion) along with increasing anti-PD1 infusions, but only one patient required the 278 introduction of a new antidiabetic treatment. It should be noticed that the weight of 279 patients did not either change significantly under anti-PD1 treatment. All these 280 observations are not suggestive of a direct effect of anti-PD1 on glucose metabolism. 281 The slight trend for an increasing glycaemia in patients with a preexisting glycemic 282 disorder might result from a lower ability to control glycemic changes induced by many 283 factors other than anti-PD1 treatment: impact of the tumor load on the general 284 metabolism, indirect consequences of other immune-related complication, differences 285 in dietary behavior, supportive treaments including steroids, etc. In patients with normal pretreatment glycaemia, the trend is so low that it can be considered negligible.

The limit of our work is related to its retrospective character, and the fact that we did not have systematically access to $\mathrm{HbA1c}$ results. Nevertheless, no case of AD1 could

292 be missed, and the study of blood glucose values does not suggest that we could find 293 different results with a prospective study or prospective HbA1c collection. The advantage of this cohort is that it is not biased by any selection on disease severity, age and general status. As steroids can potentially affect glycemic levels, it is important to notice that only eight patients were treated with systemic steroids to manage irAE or symptomatic cerebral oedema. 
Better determining the monitoring of asymptomatic individuals under anti-PD1 therapy has crucial cost and care implications. When investigating the relationship between asymptomatic grade 3 or higher increases in amylase and/or lipase and pancreatitis in melanoma patients who received a combination of nivolumab + Ipilimumab, Friedman and collegues [42] found only two cases of pancreatitis, representing roughly $20 \%$ of patients with grade 3 or higher amylase, or amylase lipase elevations. Our data suggest that close monitoring of glycaemia in all patients treated by anti-PD1 is useless since there was no general tendency to glycemic disorder and since AD1 cannot be anticipated from blood glucose monitoring. Furthermore, for type 2 diabetic patients, there is no reason to change the regular monitoring of their diabetes under anti-PD1 therapy. From the practical point of view, it is important to sensitize practitioners to the risk of a sudden severe ketosic decompensation in patients treated with anti-PD1, but also to inform patients on the usual symptoms, since misdiagnosis or delayed management can be fatal. HLA-genotyping before treatment may be useful to focus surveillance in patients with the HLA DRB $1{ }^{*} 03 / 04$ group. Conversely, it would not be sensible to contraindicate anti-PD1 for these patients in the context of a deadly metastatic disease, but it may be cautious to exclude these groups from adjuvant treatment with anti-PD1.

The occurrence of IRAE under anti-PD1 being potentiated by use of immunotherapy such as anti-CTLA4 antibodies, it will therefore be necessary to be particularly vigilant and reactive in patients receiving combination or sequence of anti-PD1 and other immune-active agents.

\section{REFERENCES}

[1] Robert C, Long GV, Brady B, Dutriaux C, Maio M, Mortier L, et al. Nivolumab in previously untreated melanoma without BRAF mutation. N Engl J Med 2015;372:32030. doi:10.1056/NEJMoa1412082.

[2] Weber JS, D'Angelo SP, Minor D, Hodi FS, Gutzmer R, Neyns B, et al. Nivolumab versus chemotherapy in patients with advanced melanoma who progressed after antiCTLA-4 treatment (CheckMate 037): a randomised, controlled, open-label, phase 3 trial. The Lancet Oncology 2015;16:375-84. doi:10.1016/S1470-2045(15)70076-8. [3] Robert C, Schachter J, Long GV, Arance A, Grob JJ, Mortier L, et al. Pembrolizumab versus Ipilimumab in Advanced Melanoma. New England Journal of Medicine 2015;372:2521-32. doi:10.1056/NEJMoa1503093.

[4] Bowyer S, Lorigan P. The place of PD-1 inhibitors in melanoma management. The Lancet Oncology 2015;16:873-4. doi:10.1016/S1470-2045(15)00094-7. 
338 Responses with Lambrolizumab (Anti-PD-1) in Melanoma. New England Journal of Medicine 2013;369:134-44. doi:10.1056/NEJMoa1305133.

340 [6] Friedman CF, Proverbs-Singh TA, Postow MA. Treatment of the Immune-Related Adverse Effects of Immune Checkpoint Inhibitors: A Review. JAMA Oncol 2016;2:134653. doi:10.1001/jamaoncol.2016.1051. Cutaneous, gastrointestinal, hepatic, endocrine, and renal side-effects of anti-PD-1 therapy. Eur J Cancer 2016;60:190-209. doi:10.1016/j.ejca.2016.02.025. [8] Mellati M, Eaton KD, Brooks-Worrell BM, Hagopian WA, Martins R, Palmer JP, et al. Anti-PD-1 and Anti-PDL-1 Monoclonal Antibodies Causing Type 1 Diabetes. Diabetes Care 2015;38:e137-8. doi:10.2337/dc15-0889.

[9] Hickmott L, Peña HDL, Turner H, Ahmed F, Protheroe A, Grossman A, et al. AntiPD-L1 atezolimumab-Induced Autoimmune Diabetes: a Case Report and Review of the Literature. Targ Oncol 2017:1-7. doi:10.1007/s11523-017-0480-y.

[10] Brahmer JR, Tykodi SS, Chow LQM, Hwu W-J, Topalian SL, Hwu P, et al. Safety and Activity of Anti-PD-L1 Antibody in Patients with Advanced Cancer. New England Journal of Medicine 2012;366:2455-65. doi:10.1056/NEJMoa1200694.

[11] Lowe JR, Perry DJ, Salama AKS, Mathews CE, Moss LG, Hanks BA. Genetic risk analysis of a patient with fulminant autoimmune type 1 diabetes mellitus secondary to combination ipilimumab and nivolumab immunotherapy. J Immunother Cancer 2016;4. doi:10.1186/s40425-016-0196-z.

[12] Ansari MJI, Salama AD, Chitnis T, Smith RN, Yagita H, Akiba H, et al. The programmed death-1 (PD-1) pathway regulates autoimmune diabetes in nonobese diabetic (NOD) mice. J Exp Med 2003;198:63-9. doi:10.1084/jem.20022125. [13] Kochupurakkal NM, Kruger AJ, Tripathi S, Zhu B, Adams LT, Rainbow DB, et al. Blockade of the Programmed Death-1 (PD1) Pathway Undermines Potent Genetic Protection from Type 1 Diabetes. PLOS ONE 2014;9:e89561. doi:10.1371/journal.pone.0089561.

[14] Wang J, Yoshida T, Nakaki F, Hiai H, Okazaki T, Honjo T. Establishment of NODPdcd1-/- mice as an efficient animal model of type I diabetes. Proc Natl Acad Sci USA 2005;102:11823-8. doi:10.1073/pnas.0505497102.

[15] Cernea S, Herold KC. Monitoring of antigen-specific CD8 T cells in patients with type 1 diabetes treated with antiCD3 monoclonal antibodies. Clin Immunol 2010;134:121-9. doi:10.1016/j.clim.2009.09.005.

[16] Ansari MJI, Salama AD, Chitnis T, Smith RN, Yagita H, Akiba H, et al. The programmed death-1 (PD-1) pathway regulates autoimmune diabetes in nonobese diabetic (NOD) mice. J Exp Med 2003;198:63-9. doi:10.1084/jem.20022125.

[17] Hughes J, Vudattu N, Sznol M, Gettinger S, Kluger H, Lupsa B, et al. Precipitation of Autoimmune Diabetes With Anti-PD-1 Immunotherapy. Diabetes Care 2015;38:e55-7. doi:10.2337/dc14-2349.

[18] Martin-Liberal J, Furness AJ, Joshi K, Peggs KS, Quezada SA, Larkin J. Antiprogrammed cell death-1 therapy and insulin-dependent diabetes: a case report. Cancer Immunol Immunother 2015;64:765-7. doi:10.1007/s00262-015-1689-1. [19] Chae YK, Chiec L, Mohindra N, Gentzler R, Patel J, Giles F. A case of pembrolizumab-induced type-1 diabetes mellitus and discussion of immune checkpoint inhibitor-induced type 1 diabetes. Cancer Immunol Immunother 2017;66:25-32. doi:10.1007/s00262-016-1913-7.

[20] Hansen E, Sahasrabudhe D, Sievert L. A case report of insulin-dependent diabetes 
as immune-related toxicity of pembrolizumab: presentation, management and outcome. Cancer Immunol Immunother 2016;65:765-7. doi:10.1007/s00262-016-1835-4. [21] Usui Y, Udagawa H, Matsumoto S, Imai K, Ohashi K, Ishibashi M, et al. Association of Serum Anti-GAD Antibody and HLA Haplotypes with Type 1 Diabetes Mellitus Triggered by Nivolumab in Patients with Non-Small Cell Lung Cancer. Journal of Thoracic Oncology 2016;0. doi:10.1016/j.jtho.2016.12.015.

[22] Hanafusa T, Imagawa A. Fulminant type 1 diabetes: a novel clinical entity requiring special attention by all medical practitioners. Nat Clin Pract Endocrinol Metab 2007;3:36-45; quiz 2p following 69. doi:10.1038/ncpendmet0351.

[23] Surveillance WHOD of ND. Definition, diagnosis and classification of diabetes mellitus and its complications : report of a WHO consultation. Part 1, Diagnosis and classification of diabetes mellitus 1999.

[24] Ishikawa K, Shono-Saito T, Yamate T, Kai Y, Sakai T, Shimizu F, et al. A case of fulminant type 1 diabetes mellitus, with a precipitous decrease in pancreatic volume, induced by nivolumab for malignant melanoma: analysis of HLA and CTLA-4 polymorphisms. European Journal of Dermatology 2200;1. doi:10.1684/ejd.2016.2923. [25] Teramoto Y, Nakamura Y, Asami Y, Imamura T, Takahira S, Nemoto M, et al. Case of type 1 diabetes associated with less-dose nivolumab therapy in a melanoma patient. J Dermatol 2016:n/a - n/a. doi:10.1111/1346-8138.13486.

[26] Okamoto M, Okamoto M, Gotoh K, Masaki T, Ozeki Y, Ando H, et al. Fulminant type 1 diabetes mellitus with anti-programmed cell death-1 therapy. J Diabetes Investig 2016:n/a - n/a. doi:10.1111/jdi.12531.

[27] Munakata W, Ohashi K, Yamauchi N, Tobinai K. Fulminant type I diabetes mellitus associated with nivolumab in a patient with relapsed classical Hodgkin lymphoma. Int J Hematol 2017;105:383-6. doi:10.1007/s12185-016-2101-4.

[28] Miyoshi Y, Ogawa 0, Oyama Y. Nivolumab, an Anti-Programmed Cell Death-1 Antibody, Induces Fulminant Type 1 Diabetes. The Tohoku Journal of Experimental Medicine 2016;239:155-8. doi:10.1620/tjem.239.155.

[29] Gaudy C, Clévy C, Monestier S, Dubois N, Préau Y, Mallet S, et al. Anti-PD1 Pembrolizumab Can Induce Exceptional Fulminant Type 1 Diabetes. Diabetes Care 2015;38:e182-3. doi:10.2337/dc15-1331. [30] Brahmer JR, Tykodi SS, Chow LQM, Hwu W-J, Topalian SL, Hwu P, et al. Safety and Activity of Anti-PD-L1 Antibody in Patients with Advanced Cancer. N Engl J Med 2012;366:2455-65. doi:10.1056/NEJMoa1200694.

[31] Aleksova J, Lau PKH, Soldatos G, McArthur G. Glucocorticoids did not reverse type 1 diabetes mellitus secondary to pembrolizumab in a patient with metastatic melanoma. BMJ Case Reports 2016;2016:bcr2016217454. doi:10.1136/bcr-2016-217454.

[32] Imagawa A, Hanafusa T, Miyagawa J, Matsuzawa Y. A novel subtype of type 1 diabetes mellitus characterized by a rapid onset and an absence of diabetes-related antibodies. Osaka IDDM Study Group. N Engl J Med 2000;342:301-7. doi:10.1056/NEJM200002033420501.

[33] Imagawa A, Hanafusa T. Fulminant type 1 diabetes-an important subtype in East Asia. Diabetes Metab Res Rev 2011;27:959-64. doi:10.1002/dmrr.1236.

[34] Fujisawa R, Haseda F, Tsutsumi C, Hiromine Y, Noso S, Kawabata Y, et al. Low programmed cell death-1 (PD-1) expression in peripheral CD4+ T cells in Japanese patients with autoimmune type 1 diabetes. Clin Exp Immunol 2015;180:452-7. doi:10.1111/cei.12603.

[35] Moreau C, Drui D, Arnault-Ouary G, Charbonnel B, Chaillous L, Cariou B. Fulminant type 1 diabetes in Caucasians: A report of three cases. Diabetes \& Metabolism 
2008;34:529-32. doi:10.1016/j.diabet.2008.05.003.

436 [36] Lévy-Marchal C, Patterson CC, Green A, EURODIAB ACE Study Group. Europe and

437 Diabetes. Geographical variation of presentation at diagnosis of type I diabetes in

438 children: the EURODIAB study. European and Dibetes. Diabetologia 2001;44 Suppl

439 3:B75-80.

440 [37] DIAMOND Project Group. Incidence and trends of childhood Type 1 diabetes 441 worldwide 1990-1999. Diabet Med 2006;23:857-66. doi:10.1111/j.1464-

$442 \quad 5491.2006 .01925 . x$.

443 [38] Karvonen M, Viik-Kajander M, Moltchanova E, Libman I, LaPorte R, Tuomilehto J.

444 Incidence of childhood type 1 diabetes worldwide. Diabetes Mondiale (DiaMond) Project 445 Group. Diabetes Care 2000;23:1516-26.

446 [39] Erlich H, Valdes AM, Noble J, Carlson JA, Varney M, Concannon P, et al. HLA DR-DQ 447 haplotypes and genotypes and type 1 diabetes risk: analysis of the type 1 diabetes 448 genetics consortium families. Diabetes 2008;57:1084-92. doi:10.2337/db07-1331.

449 [40] Hua C, Boussemart L, Mateus C, et al. ASsociation of vitiligo with tumor response 450 in patients with metastatic melanoma treated with pembrolizumab. JAMA Dermatol 451 2016;152:45-51. doi:10.1001/jamadermatol.2015.2707.

452 [41] Eschwege E, Basdevant A, Crine A, Moisan C, Charles M-A. Type 2 diabetes 453 mellitus in France in 2012: results from the ObEpi survey. Diabetes Metab 2015;41:55454 61. doi:10.1016/j.diabet.2014.11.007.

455 [42] Friedman CF, Clark V, Raikhel AV, Barz T, Shoushtari AN, Momtaz P, et al. 456 Thinking Critically About Classifying Adverse Events: Incidence of Pancreatitis in 457 Patients Treated With Nivolumab + Ipilimumab. J Natl Cancer Inst 2017;109.

458 doi:10.1093/jnci/djw260.

459

460 


\section{Figure legends}

465

Figure 1: Whole population $(n=160)$ : Median ( $\min , \max )$ glycaemia with successive 466

467 Figure 2: Patients with normal glycaemia before treatment $(n=132)$ : evolution of 468 median (min, max) glycaemia with successive anti-PD1 infusions.

470 Figure 3: Patients with abnormal glycaemia before treatment $(n=28)$ : evolution of 471 median (min, max) glycaemia with successive anti-PD1 infusions. 